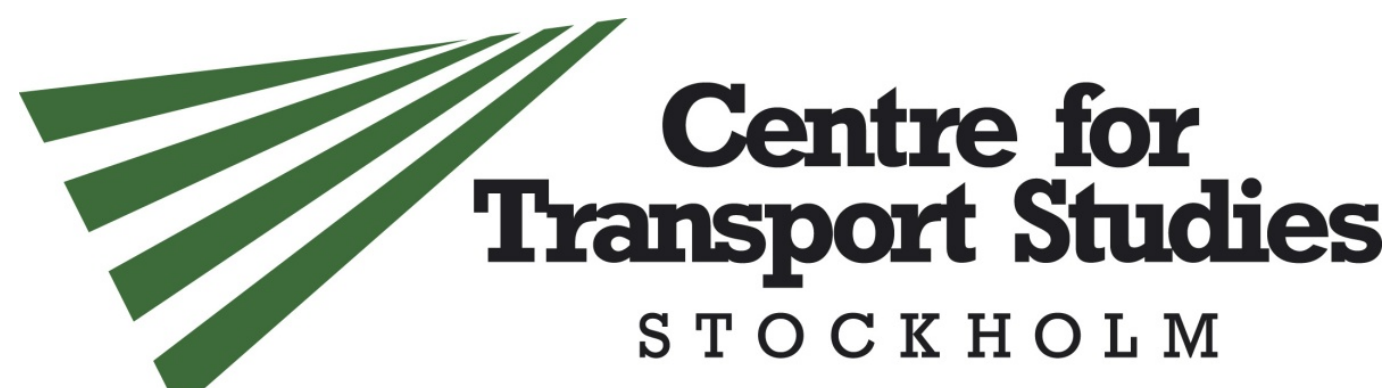

Sharing costs in Swedish road ownership associations

Sofia Grahn-Voorneveld - VTI

CTS Working Paper 2011: 6

\begin{abstract}
Usually transport systems, and roads in particular, are viewed as public goods. However, this is not always the case. In Sweden a large part of the road system is privately owned. Most of these privately owned roads are rural roads used by farmers and summer cottage owners, or used for forest transport. The roads are mainly provided by ownership associations. The motivation of this paper is the practical problem of how such an ownership association can divide the costs for the road network among the members in a "fair" way. The problem is treated from a game theoretical point of view, making use of the Shapley value. This means that the problem is associated with a game - a mathematical representation of the conflict situation. The Shapley value is a very important solution concept for cooperative games, like the game in this case. For games corresponding to this specific type of problems, it is shown that the Shapley value has excellent properties, such as being an element of the core, and being very easy to compute.
\end{abstract}

Keywords: Transport networks; Cooperative game theory

JEL Codes: H71, L91 



\title{
Sharing costs in Swedish road ownership associations
}

\author{
Sofia Grahn-Voorneveld* \\ Swedish National Road and Transport Research Institute \\ P.O.Box 55685, 10215 Stockholm, Sweden
}

\begin{abstract}
Usually transport systems, and roads in particular, are viewed as public goods. However, this is not always the case. In Sweden a large part of the road system is privately owned. Most of these privately owned roads are rural roads used by farmers and summer cottage owners, or used for forest transport. The roads are mainly provided by ownership associations.

The motivation of this paper is the practical problem of how such an ownership association can divide the costs for the road network among the members in a "fair" way. The problem is treated from a game theoretical point of view, making use of the Shapley value. This means that the problem is associated with a game - a mathematical representation of the conflict situation. The Shapley value is a very important solution concept for cooperative games, like the game in this case. For games corresponding to this specific type of problems, it is shown that the Shapley value has excellent properties, such as being an element of the core, and being very easy to compute.
\end{abstract}

*I thank Jan-Eric Nilsson for useful comments. 


\section{Introduction}

Usually transport systems, and roads in particular, are viewed as public goods. However this is not always the case. Sweden is a sparsely populated country, with large areas that are unpopulated or extremely sparsely populated. While the communities (or sometimes the government) are committed to make a road network available for use in all places covered by a city plan, there is no legal requirement to do so outside these areas.

Today's road network comprise some $419000 \mathrm{~km}$ roads. More than half, some 284 $000 \mathrm{~km}$, are privately owned. Of the privately owned roads some $150000 \mathrm{~km}$ are used for forest transport. These roads are typically not maintained between major logging events but are often open for the public. The rest of the private roads are owned by some 60 000 property owners or private road associations (samfällighetsföreningar). Some 24000 of these road associations receive state subsidies towards covering parts of the costs for construction and maintenance. The state subsidies are conditioned on that the roads are held open for public use.

The ownership associations are formed in cooperation with the National land survey of Sweden - Lantmäteriet, and the rules for membership and many other aspects are regulated by law. Any real property, within the area for the road system of the association, can be forced to join the association if the benefit from the road system is considered to be essential for the real property in question. In connection with the constitution of the road association, it is decided what share of the costs, for building and maintaining the road, each real property has to carry. The share is related to characteristics of the property, not to the actual use of the road. An obvious characteristic is the road length needed to connect a property to the public road network. Other possible characteristics are whether the real property is a summer cottage, all year around house or a farm. If the property is a forest area the share is usually related to the size of the forest area. Today the shares are decided by the road association or the National land survey of Sweden. If the road association decides the shares, they have to be approved by the National land survey to make sure that they are "reasonable". However, the National land survey does not have rigid rules for what the shares should be, only guidelines.

In transport related literature the problem of dividing costs among the users and principles for such divisions are seldom approached. Literature on club theory deals with problems similar to this one (see for instance Buchanan 1965). However, there is a significant difference; In club theory the membership of a club should be voluntary, and it must be possible to exclude non-members from using the facility. This is not the case in the situation modelled in this paper.

The motivation of this paper is the practical problem of how such a road association can divide the costs among the members in a "fair" way. The costs are of two different types. The first type are costs that are directly related to the usage of the road, i.e. the marginal cost. Even though the actual use is not measured it is possible to let every user pay roughly their own cost. However, a large part of the costs relate to building costs or maintenance costs related to weather rather than usage, for instance road damage in connection to thawing of the ground. The problem of how to divide this second type 
of costs is treated from a game theoretical point of view, making use of the Shapley value. For this purpose a new class of problems are defined - the class of road association problems. Each such problem can be associated with a cooperative game - a mathematical representation of the conflict situation. These games are related to a well known class of games - the class of airport games.

Even though fairness is a subjective property and there exist no "correct" cost allocation to the problem, some allocations are more likely to be acceptable than others. These are allocations that satisfy properties related to fairness. The Shapley value is a very important solution concept for cooperative games since it satisfies many such properties. For games corresponding to road association problems it is shown that the Shapley value can be written as a very simple expression, which makes it easy to calculate. Further it is shown that for these games the Shapley value is always an element of the core.

\section{The model}

The road system of a road association can have very different structures. To create a model to handle this some definitions from graph theory ${ }^{1}$ are used.

Definition 2.1 A graph is a pair $(V, L)$ where $V$ is a non-empty set of elements called nodes (or vertices or points), and $L$ is a finite set of unordered pairs of distinct elements of $V$ called links (or edges)

\section{$\triangleleft$}

Figure 1: The connection between a pair of nodes is a link.

Note that a graph is a general structure, allowing cycles, as in figure 2.

In this specific context the following definition is used.

Definition 2.2 A root is a node that connects the road association network to the public road network or other facility of importance to the members of the road association, such as a harbour.

\footnotetext{
${ }^{1}$ In mathematics, graph theory is the study of mathematical structures used to model pairwise relations between objects from a certain collection.
} 




Figure 2: A graph

It is possible that the graph corresponding to a road association network has several roots.

The problem for the road association is to divide the costs of its road network. The costs are of two different types. The first type of cost is directly related to how much a road is used. Since the actual use is not measured, the real properties are classified depending on their expected usage. The set $N=\{1, \ldots, n\}$ of members of the road association is divided into $k$ groups with $n_{1}, \ldots, n_{k}$ members in each group respectively. Each group $g_{i}$ is associated with a maintenance cost $\alpha_{i}$, which is the expected maintenance cost caused by a member of this group. Since this type of cost is independent of the needs or deeds of other actors, it is reasonable that every real estate carries its own costs, i.e. real estate $i$ pay $\alpha_{i}$.

The second type of costs are fixed with respect to the usage. Instead they are related to the different needs for sophistication of the road network. An obvious sophistication factor is the road length. Example of other sophistication factors are bearing capacity and winter standard. To be able to handle the second type of costs a new class of problems is defined.

Definition 2.3 A road association problem is a tuple $(N, T, F, f, c)$ consisting of

- a finite set of members $N \subset \mathbb{N}$;

- a graph $T$ consisting of nodes, roots and a set $L$ of links.

- a set $F \subset \mathbb{N}$ of sophistication factors;

- a function $f: N \rightarrow 2^{L \times F}$ specifying for every member which links he needs with what sophistication factor;

- a function $c: L \times F \rightarrow \mathbb{R}_{+}$specifying the cost of each link with respect to every sophistication factor.

The set $2^{L \times F}$ denotes the set of all possible combinations of links and sophistication levels. A player who does not need a specific link use this link with a sophistication level associated with a cost equal to zero. 
Example 2.4 Consider a road association with the following graph:



Figure 3: The graph

The graph has three links $A, B$ and $C$. Link $A$ is needed by all five real estates of the road association, although real estate 1 needs the link with a lower sophistication level than the rest of the real estates. Link $B$ is needed by real estates 2 and 3 . Real estates i.e. 1, 4 and 5 do not need this link, and therefore use the link with a sophistication level associated with a cost equal to zero. Link $C$ is needed by real estates 4 and 5 , but not by real estates 1, 2 and 3 which therefore use it with a sophistication level associated with a cost equal to zero.

It is possible to order the users/real properties in terms of their needs with respect to every sophistication factor. Accommodating a user with a certain need allows accommodating all users with lower needs at no extra cost. This type of problem is known as an airport problem, since it originally was phrased as the problem of financing a landing strip. Every airport problem can be associated with a coalitional form game called an airport game.

A cooperative cost game is a tuple $\langle N, c\rangle$ where $N=\{1, \ldots, n\}$ is the set of players. The set of all possible coalitions of players in $N$ is denoted by $2^{N}=\{S \mid S \subseteq N\}$. The function $c: 2^{N} \rightarrow \mathbb{R}$ is called the characteristic cost function of the game, and assigns to each coalition $S$ a cost $c(S) \in \mathbb{R}$, with $c(\varnothing)=0$.

Definition 2.5 Airport game: Let $N$ denote the set of players. The set $N$ is split into $k$ groups of players $g_{1}, \ldots, g_{k}$ with $n_{1}, \ldots, n_{k}$ players in each group respectively. Each group is assigned a non-negative number $b_{1}, \ldots, b_{k}$. The airport game corresponding to $g_{1}, \ldots, g_{k}$ and $b_{1}, \ldots, b_{k}$ is the cooperative cost game $\langle N, c\rangle$, where $N$ is the set of players $N=\cup_{i=1}^{k} g_{i}$, and the cost function $c$ is defined by

$$
c(S)=b_{1}+\ldots+b_{m(S)}
$$

for every $S \subseteq N$, where $m(S)=\max \left\{m: S \cap g_{m} \neq \emptyset\right\}$. 
An airport game is a cost game for the financing of one facility where the needs of the coalitions are linearly ordered. The groups $g_{1}, \ldots, g_{k}$ are groups of players with different desires for sophistication levels (originally length of landing strip). Every $b_{l}$ represents the extra building cost needed to adjust a facility, used by the less sophisticated players of groups $g_{1}, \ldots, g_{l-1}$, to be used also by the more sophisticated players in group $g_{l}$.

For a road association problem every link of the road network corresponds to an airport game for every sophistication factor. Let $\widetilde{G_{i j}}$ be the airport game corresponding to link $i$ and sophistication factor $j \in F$. The members of the road association are divided into $k(i j)$ groups of players $g_{1}^{i j}, \ldots, g_{k(i j)}^{i j}$ with $n_{1}^{i j}, \ldots, n_{k(i j)}^{i j}$ players in each group respectively. Every group $g_{q}^{i j}$ is associated with a cost $b_{q}^{i j}$ which is the cost to raise the standard of the road from what is needed by group $g_{q-1}^{i j}$ to the standard needed by group $g_{q}^{i j}$. Members of the road association who do not need this link has sophistication level zero associated with a cost equal to zero

Definition 2.6 Road association game: Every road association problem corresponds to a game $G=\langle N, c\rangle$ which is the sum of a finite set of airport games, where each link corresponds to an airport game for every sophistication factor. The cost function $c$ of the game $G$ is defined by

$$
c(S)=\sum_{i \in L, S \cap N_{i} \neq \varnothing} \sum_{j \in F}\left(b_{1}^{i j}+\ldots+b_{m_{i j}(S)}^{i j}\right)
$$

for every $S \subseteq N$, where $m_{i j}(S)=\max \left\{m: S \cap g_{m}^{i j} \neq \emptyset\right\}$, i.e. $g_{m_{i j}(S)}$ is the group with the highest sophistication level containing members of $S$.

This means that the $c(S)$ is the cost needed to build/maintain the road network with a sophistication level, with respect to all sophistication factors, so that all members of $S$ can use the road network.

Example 2.7 Consider the graph in example 2.9. Assume that the real properties 2,3,4 and 5 are summer cottages while real property 1 is a farm with need for higher bearing capacity. The problem has two sophistication factors; the road itself (factor 1) and bearing capacity (factor 2). Each link therefore corresponds to two different airport games, one for each sophistication factor. For every game the players are divided into different groups depending on their need for sophistication level. Every such group is associated with a cost as described above. The costs for this example are given in the table below.

The game $A_{1}$ relates to the costs for building and/or maintaining link $A$ with a normal standard. The members of the road segment either use part of the link (player 1) or use the whole link (players 2,3,4,5). These two groups therefore corresponds to different sophistication levels, with respect to road length. The groups are denoted by $g_{1}^{A 1}=\{1\}$ and $g_{2}^{A 1}=\{2,3,4,5\}$. The set $g_{1}^{A 1}$ (Player 1$)$ is of a lower sophistication level than the rest of the players, who need the whole road segment of link $A$. The cost $b_{1}^{A 1}$ is the cost for building/maintaining the lowest sophistication level, i.e. the road section used by player 
1. The $\operatorname{cost} b_{2}^{A 1}$ is the cost for raising the road standard to sophistication level 2 , i.e. the costs for the rest of the road segment.

The game $A_{2}$ relates to the costs for sophistication factor 2 - bearing capacity. Player 2, 3, 4 and 5 need no extra bearing capacity than what is included in normal road standard covered by sophistication factor 1 . The cost associated with their sophistication level is therefore zero. Hence, $g_{1}^{A 2}=\{2,3,4,5\}$ is associated with the cost $b_{1}^{A 2}=0$. Player 1 has a need for a higher sophistication level. Thus $g_{2}^{A 2}=\{1\}$ is associated with the cost $b_{2}^{A 2}$ which is the cost to maintain the road section of link $A$ with the extra bearing capacity needed by player 1 .

The group division and costs for the games corresponding to links $B$ and $C$ are shown in the table below:

\begin{tabular}{|l|l|l|l|l|l|l|}
\hline Game & $b_{1}^{i j}$ & $b_{2}^{i j}$ & $g_{1}^{i j}$ & $g_{2}^{i j}$ & $n_{1}^{i j}$ & $n_{2}^{i j}$ \\
\hline$A_{1}$ & 1 & 2 & $\{1\}$ & $\{2,3,4,5\}$ & 1 & 4 \\
\hline$A_{2}$ & 0 & 2 & $\{2,3,4,5\}$ & $\{1\}$ & 4 & 1 \\
\hline$B_{1}$ & 0 & 4 & $\{1,4,5\}$ & $\{2,3\}$ & 3 & 2 \\
\hline$B_{2}$ & 0 & - & $\{1,2,3,4,5\}$ & - & 5 & - \\
\hline$C_{1}$ & 0 & 2 & $\{1,2,3\}$ & $\{4,5\}$ & 3 & 2 \\
\hline$C_{2}$ & 0 & - & $\{1,2,3,4,5\}$ & - & 5 & - \\
\hline
\end{tabular}

where $n_{l}^{i j}$ is the number of members in group $g_{l}^{i j}$. The road association game $G$, corresponding to this road association problem, is the sum of the above 6 airport games. $\triangleleft$

\section{Dividing the costs}

The problem that every road association face is how to divide the costs for its road network in a "fair" way among the members. A road association game $G$, corresponding to a road association problem, describes the costs that each coalition would face if the coalition were to act separate from the players outside this coalition. Now an allocation rule allocating the costs among the players has to be chosen.

A very important solution concept for transferable utility games is the Shapley value, which has excellent properties and has been applied successfully in cost allocation problems (see Shapley (1953), Tijs and Driessen (1986), Young (1994), Moulin and Schenker (1996) and Fragnelli et al. (1999)).

The Shapley value was introduced by Shapley (1953) and can be characterized by four properties. Somewhat informally:

- efficiency, i.e. no resources are wasted;

- anonymity, i.e. two identical players are treated equally;

- dummy property, i.e. a player with a constant marginal contribution to every coalition of which he is a member, is allocated this constant. 
- additivity, i.e. the solution of the sum of two games, is the sum of the solution to the two games.

The Shapley value is the only solution concept satisfying all four of these axioms.

In this context the Shapley value assigns to each player the average marginal cost contribution that the player makes to each of the coalitions to which he belongs.

Let $\Phi_{i}(c)$ denote the Shapley value of a cost game $\langle N, c\rangle$, then the Shapley value can be written as

$$
\Phi_{i}(c)=\sum_{S: i \notin S} \frac{|S| !(n-1-|S|) !}{n !}(v(S \cup\{i\})-v(S)) \text { for player } i \in N
$$

In a practical environment it is extremely important that the solution allocation can be computed easily. For an airport game the Shapley value can be written as

$$
\Phi_{i}(c)=\sum_{l=1}^{q(i)} \frac{b_{l}}{\sum_{p=l}^{k} n_{p}} \text { for all } i \in N
$$

(see Littlechild and Owen (1973)). For a road association game, the Shapley value can be written as the sum of the Shapley values of the link games of the corresponding road association problem. This follows from the additivity property of the Shapley value. Thus:

$$
\Phi_{k}(c)=\sum_{i \in L, k \in N_{i}} \sum_{j \in F} \sum_{l=1}^{q(i)} \frac{b_{l}^{i j}}{\sum_{p=l}^{k(i j)} n_{p}} \text { for all } k \in N
$$

Example 3.1 Using the same road association as in example 2.7 the corresponding road association game $G$ is the sum of 9 airport games. Using expression (1) we get

\begin{tabular}{|l|l|l|l|l|l|}
\hline Game & player 1 & player 2 & player 3 & player 4 & player 5 \\
\hline$A_{1}$ & $\frac{1}{5}$ & $\frac{1}{5}+\frac{2}{4}=\frac{14}{20}$ & $\frac{1}{5}+\frac{2}{4}=\frac{14}{20}$ & $\frac{1}{5}+\frac{2}{4}=\frac{14}{20}$ & $\frac{1}{5}+\frac{2}{4}=\frac{14}{20}$ \\
\hline$A_{2}$ & 2 & 0 & 0 & 0 & 0 \\
\hline$B_{1}$ & 0 & $\frac{4}{2}=2$ & $\frac{4}{2}=2$ & 0 & 0 \\
\hline$B_{2}$ & 0 & 0 & 0 & 0 & 0 \\
\hline$C_{1}$ & 0 & 0 & 0 & $\frac{2}{2}=1$ & $\frac{2}{2}=1$ \\
\hline$C_{2}$ & 0 & 0 & 0 & 0 & 0 \\
\hline Sum & $\frac{13}{5}$ & $\frac{27}{10}$ & $\frac{27}{10}$ & $\frac{17}{10}$ & $\frac{17}{10}$ \\
\hline
\end{tabular}

resulting in the Shapley value $\left(\frac{13}{5}, \frac{27}{10}, \frac{27}{10}, \frac{17}{10}, \frac{17}{10}\right)$.

An intuitive property of a solution concept is that it should allocate the profit in such a way that no player or coalition of players would have been better of by them selves. The set of such allocations is called the core.

Definition 3.2 The core of the cost game $\langle N, c\rangle$ is the set 


$$
C(c):=\left\{x \in \mathbb{R}^{N} \mid \sum_{i=1}^{n} x_{i}=c(N) \text { and } \sum_{i \in S} x_{i} \leq c(S) \text { for all } S \in 2^{N} \backslash \varnothing\right\}
$$

It is well known that the Shapley value of an airport game lies in the core of the game. See for instance Thomson (2007). Due to the additivity of the Shapley value also the Shapley value of a road association game lies in the core.

\section{Conclusion}

In this paper the problem of dividing costs for a road association network over its members is addressed. Since the membership of the road ownership association is not voluntary the problem cannot be viewd as an optimization problem nor can it be adressed by club theory since the membership of a club should be voluntary and it must be possible to exclude non-members from using the club-facility. Neither of which is the case here.

The costs of a road ownership associations are of two different types. The first type are costs that are directly related to the usage of the road, i.e. the marginal cost. Even though the actual use is not measured it is possible to let every user pay roughly their own costs. However, a large part of the costs are not related to the actual usage. The problem of how to divide this second type of costs is treated from a game theoretical point of view. The problem has a structure where it is possible to order the users in terms of their needs, with respect to every sophistication factor. Accommodating a user with a certain need allows accommodating all users with lower needs at no extra cost. This type of problem is known as an airport problem. Every airport problem can be associated with a coalitional form game called an airport game. Therefore the problem of dividing the second type of costs over the members of the road association is modelled as a game, which is a sum of a finite number of airport games.

The Shapley value is one of the most important solution concepts for cooperative games. The Shapley value of a cost game assigns to each player the marginal cost contribution that the player makes to each of the coalitions to which he belongs. The marginal cost contribution in this context is the cost the player adds to the coalition if he has a higher need for sophistication then the rest of the players of the coalition, i.e. the sophistication has to increase so that also this player can use the road network. Apart from the nice properties that defines the Shapley value, the Shapley value always lies in the core of a game corresponding to a road association problem. Further, the Shapley value for games corresponding to road association problems can be written as a simple expression. This makes it very easy to calculate the Shapley value for this type of problem. The Shapley value therefore is a very appealing solution concept for road association problems.

\section{References:}

Buchanan J. M. (1965): "An economic theory of clubs", Economica 32(1): 1-14. 
Littlechild S. And Owen G. (1973): "A Simple Expression for the Shapley Value in a Special Case", Management Science 20:370-372.

Fragnelli V., García-Jurado I., Norde H., Patrone F. And Tijs S. (1999): "How to share railways infrastructure costs?", In Game Practice: Contributions from Applied Game Theory, Kluwer Academic Press, Dordrecht.

Moulin H. And Schenker S. (1996): "Strategyproof Sharing of Submodular Access Costs: Budget Balance versus Efficiency". Mimeo.

Shapley L. S. (1953): "A Value for n-Person Games" In: Contributions to the Theory of Games II. H Kuhn and AW Tucker (eds), pp 307-317. Princeton University Press.

Tijs S. And Driessen T. (1986): Game Theory and Cost Allocation Problems. Management Science 32:1015-1028.

Thomson W. (2007): "Cost allocation and airport problems", Working Paper No. 538, University of Rochester.

Young P. (1994): Cost Allocation. In Handbook of Game Theory (vol 2). R. J. Aumann and S Hart (eds), pp 1193-1235. North-Holland. 